\title{
Gabapentin and cognition: a double blind, dose ranging, placebo controlled study in refractory epilepsy
}

\author{
John Paul Leach, Jacqueline Girvan, Audrey Paul, Martin J Brodie
}

Epilepsy Unit, University Department of Medicine and Therapeutics, Western Infirmary, Glasgow, Scotland

J P Leach

J Girvan

A Paul

M J Brodie

Correspondence to:

Professor M J Brodie,

Epilepsy Unit, Department

of Medicine and

Therapeutics, Western

Infirmary, Glasgow

G11 6NT, Scotland, UK.

Received 2 July 1996

and in revised form

23 September 1996
Accepted 30 October 1996
(3.8), and 8.6 (3.3) $\mathrm{mg} / \mathrm{l}$ respectively. The drug had no effect on composite psychomotor and memory scores; nor was there alteration in any self assessment subscore. The mean drowsiness $(P=0.03)$ score was higher during treatment with $2400 \mathrm{mg}$ GBP daily compared with matched placebo. Composite psychomotor $(r=-0.47$, $P<0.01)$, tiredness $(r=0.42, P<0.01)$, and side effect $(r=0.61, P<0.001)$ scores correlated significantly with seizure frequency but not with GBP dose.

Conclusion-GBP is a well tolerated and effective antiepileptic drug which had no measurable effect on cognition but did produce sedation at the highest dose. This study also supports the suggestion that seizures can cause cognitive impairment.

(F Neurol Neurosurg Psychiatry 1997;62:372-376)

Keywords; gabapentin; cognition; epilepsy.

Gabapentin (GBP) is a new antiepileptic drug that was licensed for use as add on therapy in the United Kingdom in $1993 .{ }^{1}$ Its mode of action is still uncertain, ${ }^{2}$ but clinical trials have shown it to be well tolerated and effective. ${ }^{35}$ Side effects reported with GBP are transient and minor, the most common being somnolence, dizziness, ataxia, and fatigue. ${ }^{6}$ We have shown previously that antiepileptic drugs can alter cognitive function in a dose related manner. ${ }^{7-11}$ If newer antiepileptic drugs such as GBP are to attain a useful place in the therapeutic armamentarium, they must be at least as well tolerated as the older agents. ${ }^{12-13}$ The aim of this study was to assess the efficacy and tolerability of different doses of GBP in patients with refractory partial seizures, with particular reference to cognitive function.

\section{Methods}

Twenty seven patients were recruited from the epilepsy unit at the Western Infirmary in Glasgow (table 1). All had epilepsy refractory to one or two antiepileptic drugs, the doses of which had remained unchanged for at least three months before recruitment. Seizures were partial with or without secondary generalisation. All patients reported at least four per month for the previous three months. They had a history of good compliance with medication, and were able to document their seizures. 
Details of the protocol were approved by the local ethics committee and the patients gave informed, written consent to their participation.

\section{PROTOCOL}

The study was a double blind, random order, crossover comparison of three sequential doses of GBP $(400 \mathrm{mg}, 600 \mathrm{mg}$, and $800 \mathrm{mg}$ all thrice daily) with a similar number of tablets of matched placebo. One month after a screening visit, patients were entered into the first 12 week treatment period. Psychomotor and cognitive testing were carried out every four weeks, before increasing the dose of GBP or placebo. After the three dose levels had been tested, patients entered a four week washout before commencing the second treatment phase (GBP or placebo). Patients were given individual appointment times. Each visit was at a similar time of day to minimise variability in drug concentrations at the time of testing. Compliance was checked by a drug history and tablet count at each visit.

\section{NEUROPYSCHOLOGICAL TESTING}

A total of 10 tests was given at each four weekly visit. These included four psychomotor tests and six tests of memory. Tests of subjective wellbeing comprised the side effects activities and life satisfaction (SEALS) questionnaire, a side effect score, and a visual analogue scale for drowsiness.

All psychomotor tests were performed at every visit. The decision time was the time taken to respond to a light by removing the finger from the base button and the movement time that taken to move the finger from the base button to extinguish the light in a choice reaction time task. The mean of 30 trials was recorded for both using the Leeds psychomotor tester. In the threshold detection test, an array of small rectangles was displayed on an Apple IIe computer screen. After a brief time, an extra rectangle was added. The patient was required to indicate which it was. The threshold was the minimum time gap between the presentation of the array and the additional stimulus that the patient required to perceive that an extra rectangle had been added. The Stroop test assessed the patient's decision making and flexibility. Patients were given a card with a list of words printed in inks of varying colours. They were then asked to go through the list, stating the colour of ink used for each word, ignoring the meaning of the word. The number of colours stated correctly within two minutes of starting formed the basis of the score. Composite psychomotor scores were calculated as previously described. ${ }^{9}$ This involved subtraction of the mean and division by the standard deviation (SD) to give each assessment a mean of zero and an SD of 1 . Addition of each score then provided a composite score which gave equal weighting to each test.

All memory tests except for the paired associated learning, which was not undertaken at baseline, were performed at every visit. The forward digit span measured the maximum number of digits the patient could recall immediately after oral presentation. Patients were allowed two trials at each level. The backward digit span measured the maximum number of digits that the patient could recall immediately after oral presentation. Both digit span tests were discontinued when two were failed at the same level. The forward visual span measured the maximum number of squares correctly reproduced in sequence as shown by the examiner. The backward visual span assessed the maximum number of squares correctly reproduced in reverse order to that shown by the examiner. The visual span tests were terminated when two consecutive tests were failed. The paired associate learning test gave a score up to 18 depending on the number of attempts required by the patient to name three sets of correctly paired words. Composite memory scores were calculated in a similar manner to the composite psychomotor scores, providing a figure that gave equal weighting to each test. ${ }^{9}$

The SEALS questionnaire involved 50 questions that assessed subjective feelings of cognitive slowing, dysphoria, irritability, fatigue, and worry. ${ }^{14}$ The period under assessment was the seven days immediately before each clinic visit. The side effects score was constructed from patients' responses to a four point grading score for 10 adverse effects (dizziness, headache, nausea, double vision, tremor, unsteadiness, dry mouth, flushing, ankle swelling, and palpitations). Only the first six were regarded as being associated with antiepileptic drug use, the other four being controls. The combined score for the six recognised antiepileptic drug associated symptoms were obtained via simple addition as all symptoms were scored on the same four point scale. To undertake the drowsiness score, patients were asked to estimate, with the help of a visual analogue scale, their degree of alertness. The measured distance along a $10 \mathrm{~cm}$ line marked at either end "fully awake" and "nearly asleep" was recorded.

\section{DRUG ASSAYS}

Carbamazepine, sodium valproate, phenytoin, and phenobarbitone concentrations were measured by enzyme immunoassay (Emit, Syva, Palo Alto, USA). Vigabatrin was extracted from plasma into ethyl acetate, heated with dansyl chloride at high $\mathrm{pH}$ to form a fluorescent derivative, and measured by high performance liquid chromatography (HPLC) with phenyl GABA as internal standard. The interassay coefficient of variation (CV) over the range $1-100 \mathrm{mg} / 1$ was $5 \%$ and the lower limit of detection was $0.1 \mathrm{mg} / \mathrm{l}^{15}$ Lamotrigine was extracted into ethinyl acetate from plasma with $2 \mathrm{M}$ sodium hydroxide and measured by HPLC with BWA 725C (Wellcome Laboratories, UK) as internal standard. The interassay $\mathrm{CV}$ over the range up to $5 \mathrm{mg} / \mathrm{l}$ was $6 \%$ with a lower limit of detection of $0.25 \mathrm{mg} / 1 .{ }^{16}$ Concentrations of GBP were determined using HPLC with fluorimetric detection. ${ }^{17}$ The interassay and intraassay variations of this method were $3.8 \%$ and $2.6 \%$ respectively at $5 \mathrm{mg} / \mathrm{l}$, and the lower limit of detection was $1 \mathrm{mg} / 1$. 
STATISTICS

Statistical analysis was carried out with the SAS statistical package. The primary measure of seizure control was the comparison of seizure frequency during active (GBP) and placebo treatment. Seizure frequencies were normalised by log transformation before analysis of variance (ANOVA). Individual cognitive function tests were analysed using Wilcoxon signed rank tests. Composite scores for memory and psychomotor performance were constructed by summation of normalised scores for related assessments and compared using ANOVA. The five SEALS subscores, the side effect scores, and the visual analogue scale for drowsiness were explored using analysis of covariance(ANCOVA), fitting the week 0 and week 16 scores as the covariate.

All ANOVA and ANCOVA models used fitted patient, period, and treatment effects. The dose titration in the study design caused dose and length of time on treatment to be confounded in the analysis, and hence no direct comparisons were made between dose levels. The primary analysis grouped together data for all dose levels. When individual doses were evaluated this was done by comparing data for a particular dose level with data from the corresponding dose of placebo.

\section{Results}

Fourteen patients received GBP during the first phase and 13 started on placebo. One patient withdrew consent to participate in the study before visit two. Four patients did not receive all doses of both treatment phases due to onset of adverse events (one on GBP, three on placebo). Of these six withdrawals, two occurred during the later stages of the seizure treatment period, so providing seizure frequency and cognitive function data for both phases of the study. One patient could not provide complete data for seizure frequency, leaving 21 evaluable patients overall (11 GBP first, 10 placebo first).

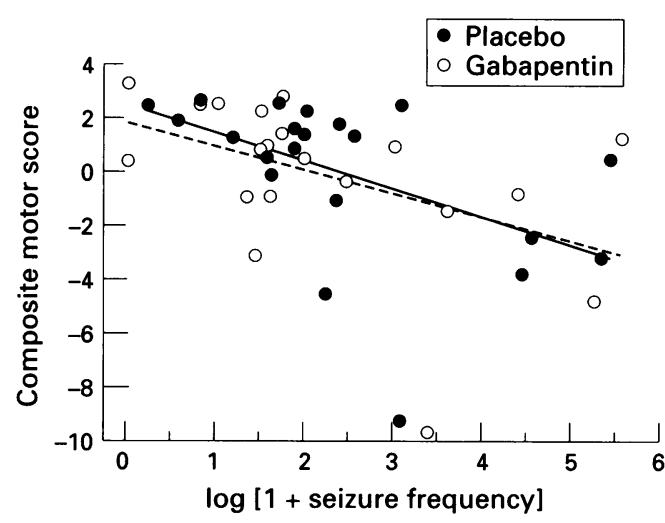

Figure 1 Correlation of composite psychomotor scores with normalised seizure frequency during treatment with adjuvant gabapentin and placebo.

\section{SEIZURE FREQUENCY}

The median monthly seizure frequency was significantly reduced by GBP treatment compared with placebo $(4.3 \vee 7, \mathrm{P}=0.02)$ throughout the active treatment period. Of the 21 patients exposed to all GBP doses, nine $(43 \%)$ had their total seizure frequency reduced by at least $50 \%$-that is, they were responders. Efficacy was greatest for the 1800 $\mathrm{mg}$ and $2400 \mathrm{mg}$ daily doses of GBP. Two patients were seizure free throughout the active treatment phase with none responding similarly to placebo. The median monthly frequency for simple partial seizures was not significantly reduced by GBP $(P=0 \cdot 80)$, although two patients $(29 \%)$ reported a reduction in excess of $50 \%$. The frequency of complex partial seizures without secondary generalisation, noted in 17 patients, was also lower on GBP, with five $(29 \%)$ documented responders. Again, however, this did not reach significance $(P=0.62)$. Ten $(59 \%)$ of 17 patients experiencing secondarily generalised seizures responded to GBP treatment, with the median monthly frequency of this type being reduced from 1 to 0.3 on GBP $(P=$

Table 2 Median psychomotor and memory scores during gabapentin and matched placebo treatment

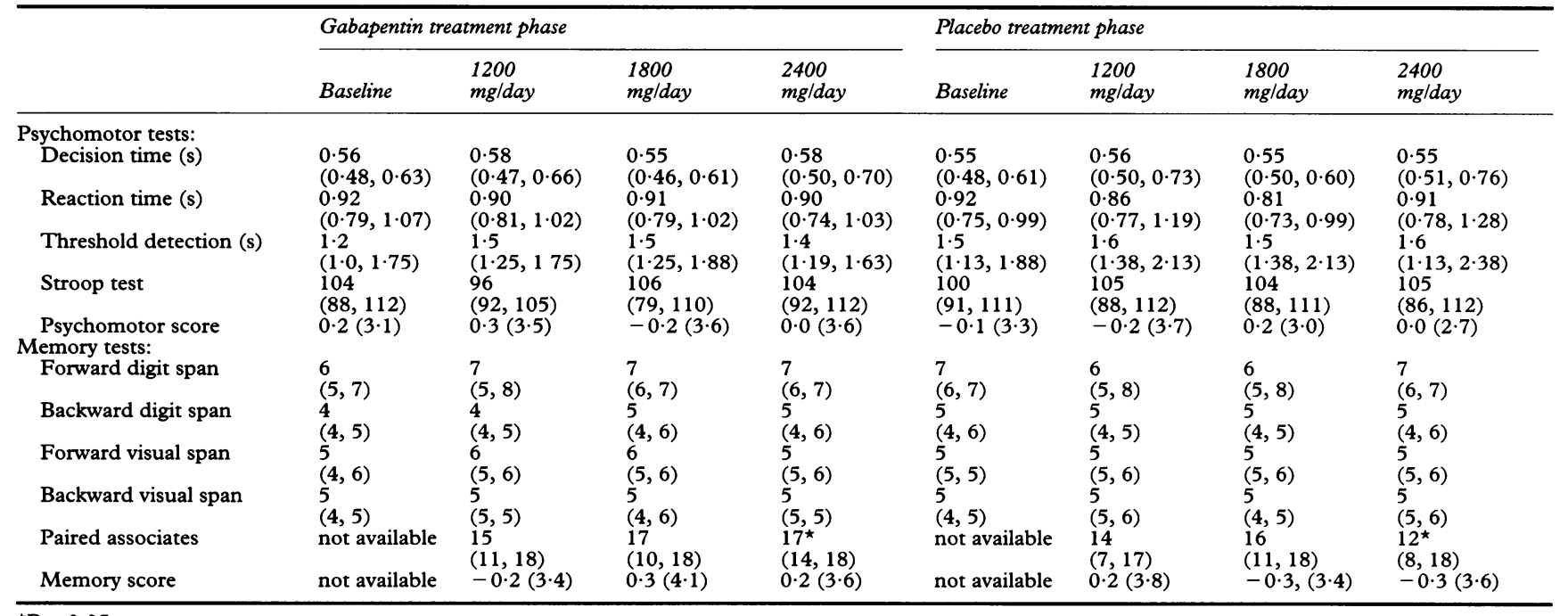

$\star \mathrm{P}<0.05$.

Figures in parentheses represent quartiles for individual tests and SDs for composite scores. 
Table 3 Mean (SD) SEALS subscores and tiredness scores at each dosage level of adjuvant gabepentin or placebo in 21 patients with refractory epilepsy

\begin{tabular}{llllllc}
\hline & $\begin{array}{l}\text { Gabapentin } \\
1200 \mathrm{mg}\end{array}$ & $\begin{array}{l}\text { Placebo } \\
1200 \mathrm{mg}\end{array}$ & $\begin{array}{l}\text { Gabapentin } \\
1800 \mathrm{mg}\end{array}$ & $\begin{array}{l}\text { Placebo } \\
1800 \mathrm{mg}\end{array}$ & $\begin{array}{l}\text { Gabapentin } \\
2400 \mathrm{mg}\end{array}$ & $\begin{array}{l}\text { Placebo } \\
2400 \mathrm{mg}\end{array}$ \\
\hline Cognition & $50(12)$ & $49(12)$ & $50(13)$ & $48(12)$ & $49(12)$ & $51(13)$ \\
Dysphoria & $16(5)$ & $17(4)$ & $17(6)$ & $16(5)$ & $17(5)$ & $17(5)$ \\
Temper & $13(3)$ & $12(3)$ & $12(4)$ & $12(3)$ & $13(4)$ & $12(3)$ \\
Fatigue & $14(4)$ & $15(3)$ & $14(4)$ & $14(4)$ & $13(3)$ & $14(4)$ \\
Worry & $10(3)$ & $10(3)$ & $11(3)$ & $10(2)$ & $10(4)$ & $10(3)$ \\
Tiredness & $35(27)$ & $44(30)$ & $47(31)$ & $33(27)$ & $\star 43(29)$ & $\star 36(27)$ \\
\hline
\end{tabular}

$\star \mathbf{P}=0.03$.

$0.01)$. There was no significant difference in response between the patients taking one or two antiepileptic drugs before introduction of GBP.

\section{NEUROPSYCHOLOGICAL TESTING}

No order effect was seen for any variable, nor was there a "learning effect" as the study progressed. Comparison of individual tests and composite psychomotor scores at each treatment level with the corresponding placebo phase failed to show any significant difference (table 2). Mean composite psychomotor scores, however, showed a negative correlation $(r=-0.47, \mathrm{P}<0.01)$ with seizure frequency (fig 1), which reached significance during treatment with both placebo $(r=-0.40, \mathrm{P}=$ $0.02)$ and GBP $(r=-0.45, P=0.04)$.

The paired associate learning test was improved on $2400 \mathrm{mg}$ GBP daily $v$ placebo. No other memory test showed a difference between GBP and placebo at any dose. There was no difference in composite memory scores between GBP and placebo treatment (table 2). No correlation was found between composite memory scores and seizure frequency during treatment with either placebo or GBP.

None of the five SEALS subscores was sig-

Figure 2 Correlation of tiredness scores with normalised seizure frequency during treatment with adjuvant gabapentin and placebo.

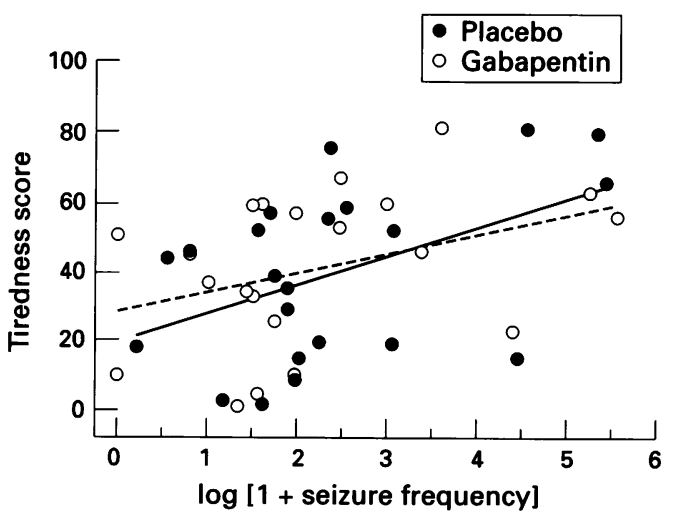

Figure 3 Correlation of side effect scores with normalised seizure frequency during treatment with adjuvant gabapentin and placebo. nificantly affected by GBP even at the highest dose, although the increases in scores for cognition and fatigue (table 3 ) narrowly failed to reach significance ( $P=0.06$ and 0.08 respectively). The mean tiredness score (table 3 ) was significantly increased only during treatment with $2400 \mathrm{mg} /$ day GBP compared with the corresponding placebo phase $(P=0.03)$. There was a significant correlation $(r=0.42$, $P<0.01)$ between the degree of tiredness reported and seizure frequency (fig 2), which reached significance during placebo treatment $(r=0.47, \mathrm{P}=0.03)$ and narrowly failed to do so with GBP $(r=0.39, P=0.08)$.

\section{ADVERSE EVENTS}

Five patients withdrew because of the onset of adverse events, four during the placebo treatment phase, and one on GBP. Nineteen patients (79\%) reported 47 adverse events on GBP, compared with 15 patients (63\%) reporting 30 adverse events on placebo. The side effect scores showed a significant difference compared with placebo while on the highest dose of GBP only $(P=0.006)$. There was a strong correlation between the side effect score and seizure frequency $(r=0.61$, $\mathrm{P}<0.001$; fig 3) during both placebo $(r=$ $0.72, P=0.001)$ and GBP treatment $(r=$ $0.50, P=0.02)$.

\section{DRUG CONCENTRATIONS}

Mean (SD) plasma concentrations of GBP at 1200,1800 , and $2400 \mathrm{mg} /$ day were $4 \cdot 7(2 \cdot 6)$, $6.8(3.8)$, and $8.6(3.3) \mathrm{mg} / \mathrm{l}$ respectively. Individual GBP concentrations did not correlate significantly with any cognitive test or composite score. There were no changes in other antiepileptic drug concentrations after GBP treatment (data not shown).

\section{Discussion}

Cognitive function is an important issue in the treatment of epilepsy. Many patients complain of impaired memory and slowed thinking while receiving long term antiepileptic drug therapy, and the vast literature on psychomotor and memory testing in epileptic patients is testimony to attempts to quantify the extent of any drug related cognitive impairment. ${ }^{12-13}$ Delineation of the deleterious effects of antiepileptic drugs on higher mental processes is vital. If the newer agents are to gain an enduring place in the therapeutic armamentarium, they will need to be at least as well tolerated as the established antiepileptic drugs.

Despite the refractory nature of the patient cohort, the seizure response rates were better than those in the other efficacy studies, a possible consequence of the higher top dose employed..$^{3-5}$ As with other studies, the effect on secondarily generalised seizures was greater than on other seizure types. ${ }^{2}$ The lack of pharmacokinetic interaction with other antiepileptic drugs was also confirmed. ${ }^{18-19}$

At doses high enough to produce a significant reduction in seizure frequency, we failed to show any effect of GBP on psychomotor or memory impairment, in keeping with previous 
reports of its good clinical tolerability. ${ }^{6}$ However, scoring on the visual analogue scale for drowsiness was significantly higher at the top GBP dose compared with placebo. At this dose, too, side effects were more likely to be reported. Other studies have shown that tolerance can develop to fatigue and drowsiness with carbamazepine and vigabatrin. ${ }^{1120}$ Were the highest dose levels to be observed for longer, perhaps this phenomenon would have been found here. Although multiple comparisons may provide isolated significant results, the fact that the composite scores measured closely allied symptoms suggests that these findings may be clinically as well as statistically significant.

The significant correlation between seizure frequency and composite psychomotor, tiredness, and side effect scores is interesting. Whether this reflects impairment of an innately more damaged brain or is secondary to the seizures themselves is difficult to assess. That the correlation between seizure frequency and these variables was much stronger than that with GBP dosage and concentration does imply, however, that optimal improvement in quality of life in epileptic patients can be gained by reducing seizure frequency with drugs that do not impair cognition.

Our grateful thanks go to Mrs Moya Dewar for typing the manuscript and to Parke-Davis for supporting the study.

1 Chadwick D. Gabapentin. Lancet 1994;343:89-91.

2 McLean MJ. Gabapentin. Epilepsia 1995;36(suppl 2): 73-86.

3 UK Gabapentin Study Group. Gabapentin in partial epilepsy. Lancet 1990;335:1114-7.

4 US Gabapentin Study Group. Gabapentin as add-on therapy in refractory epilepsy: a double-blind, placebo-con- trolled, parallel group study. Neurology 1993;43:2292-8.

5 Anhut H, Ashman P, Feuerstein TJ, et al. Gabapentin as add-on therapy in patients with partial seizures: a doubleblind, placebo-controlled study. Epilepsia 1994;35: 795-801.

6 Dichter MA, Brodie MJ. New antiepileptic drugs. N Engl $\mathcal{F}$ Med 1996;334:1583-90.

7 Brodie MJ, McPhail E, Macphee GJA, Larkin JG, Gray JMB. Psychomotor impairment and anticonvulsant therapy in adult epileptic patients. Eur f Clin Pharmacol 1987; 31:655-60.

8 Gillham RA, Williams N, Wiedmann KD, Butler E, Larkin JG, Brodie MJ. Concentration effect relationships with carbamazepine and its epoxide on psychomotor and cogcarbamazepine and its epoxide on psychomotor and cog-
nitive function in epileptic patients. $₹$ Neurol Neurosurg nitive function in epileptic
Psychiatry 1988;51:929-33.

9 Gillham RA, Williams N, Wiedmann KD, Butler E, Larkin JG, Brodie MJ. Cognitive function in adult epileptic patients established on anticonvulsant monotherapy. Epilepsy Res 1990;7:219-25

10 Gillham RA, Read CL, McKee PJW, Larkin JG, Brodie MJ. Cognitive function in adult epileptic patients on long-term sodium valproate. 7 Epilepsy $1991 ; 4: 205-10$.

11 Gillham RA, Blacklaw J, McKee PJW, Brodie MJ. Effect of vigabatrin on sedation and cognitive function in patients with refractory epilepsy. $₹$ Neurol Neurosurg Psychiatry 1993;56:1271-5.

12 Vermeulen J, Aldenkamp AP. Cognitive side-effects of chronic antiepileptic drug treatment: a review of 25 years of research. Epilepsy Res 1995;22:65-95.

13 Kalviainen R, Aikia M, Riekkinen PI. Cognitive adverse effects of antiepileptic drugs. CNS Drugs 1996;5:358-68.

14 Brown SW, Tomlinson LL. Anticonvulsant side-effects: a Brown SW, Tomlinson LL. Anticonvulsant side-effects: a
self-report questionnaire for use in community surveys. Br f Clin Pract 1984;(suppl 18): 147-9.

15 McKee PJW, Blacklaw J, Friel E, Thompson GG, Gillham RA, Brodie MJ. Adjuvant vigabatrin in refractory epilepsy: a ceiling to effective dosage in individual patients? Epilepsia 1993;34:937-43.

16 Kilpatrick ES, Forrest G, Brodie MJ. Concentration-effecttoxicity relationships with lamotrigine-a prospective study. Epilepsia 1996;37:534-8.

17 Forrest G, Sills GJ, Leach JP, Brodie MJ. Determination of gabapentin in plasma by high performance liquid chromatography. F Chromatogr B Biomed Appl 1996;681: 421-5.

18 Hooper WE, Kavanagh MC, Herkes GK, Eadie MJ. Lack of a pharmacokinetic interaction between phenobarbitone a pharmacokinetic interaction between phenobarbitone

19 Radulovic LL, Wilder BJ, Leppik IE, et al. Lack of interaction of gabapentin with carbamazepine or valproate. tion of gabapentin with

20 Larkin JG, McKee PJW, Brodie MJ. Rapid tolerance to acute psychomotor impairment with carbamazepine in epileptic patients. Br $\mathcal{F}$ Clin Pharmacol 1992;33:111-4. 